\title{
Escala de Satisfacción Laboral a Partir de Factores Socioculturales y Ergoambientales para los Docentes de las Instituciones de Educación Superior en México
}

\section{JOB SATISFACTION SCALE FROM SOCIOCULTURAL AND ENVIRONMENTAL ERGONOMIC FACTORS FOR TEACHERS IN HIGHER EDUCATION INSTITUTIONS IN MEXICO}

Maria Teresa Escobedo Portillo', Luis Cuautle Gutiérrez², Aurora Maynez Guadarrama ${ }^{3}$, Virginia Estebané Ortega ${ }^{4}$

1. 3. 4. Universidad Autónoma de Ciudad Juárez. Chihuahua. México.

2. Universidad Popular Autónoma del Estado de Puebla. México.

\begin{abstract}
RESUMEN
Se realiza una búsqueda exhaustiva de las variables pertenecientes a la Satisfacción Laboral, los Factores Socioculturales y los Factores Ergoambientales. Se examinan cuatro instrumentos de medición de la Satisfacción Laboral validados en el sector educativo de varios países, seleccionando las variables más relevantes para el caso de estudio. Se presenta la operacionalización de las variables del instrumento específico conformado por 32 items. Se valida en una prueba piloto de 26 encuestas con un nivel de confianza del 95\%, obteniendo un valor Alpha de Crombach de 0,941. Se recomienda aplicarlo en las Instituciones de Educación Superior en México, tanto privadas como públicas y observar los resultados.
\end{abstract}

(Escobedo M, Gutiérrez L, Maynez A, Ortega V, 2014. Escala de Satisfacción Laboral a Partir de Factores Socioculturales y Ergoambientales para los Docentes de las Instituciones de Educación Superior en México. Cienc Trab. Sep-Dic; 16 [51]: 177-184).

Palabras clave: SATISFACCIÓN LABORAL, FACTORES SOCIALES, FACTORES CULTURALES, ERGONOMÍA AMBIENTAL, VALIDEZ Y CONFIABILIDAD.

\section{ABSTRACT}

An exhaustive search of the variables belonging to the Job Satisfaction, Sociocultural Factors and environmental ergonomic factors is performed. Four instruments measuring job satisfaction validated in the education sector in several countries, selecting the most relevant variables to the case study are examined. The operationalization of the variables of the specific instrument conformed 32 by items is presented. It is validated in a pilot test of 26 surveys with a level of confidence of 95\%, obtaining a Cronbach Alpha value of 0,941. It is recommended to apply in Institutions of Higher Education in Mexico, both private and public and observe the results.

Key words: JOB SATISFACTION, SOCIAL FACTORS, CULTURAL FACTORS, ENVIRONMENTAL ERGONOMICS, VALIDITY AND RELIABILITY.

\section{INTRODUCCIÓN}

De manera análoga a otras organizaciones, en las Instituciones de Educación Superior (IES) el recurso humano es su base ${ }^{1}$; por ello, evaluar su Satisfacción Laboral (SL) es un factor relevante ${ }^{2}$ ya que con base en dicha valoración es posible determinar la forma en que los empleados alcanzan sus objetivos, así como las condiciones y factores que influyen en sus logros ${ }^{3}$.

En el marco del estudio de las actitudes hacia el trabajo se encuentra la SL descrita en palabras de Belfield y Harris ${ }^{4}$ como

Correspondecia / Correspondence:

Maria Teresa Escobedo Portillo

Instituto de Ingeniería y Tecnología de la UACJ.

Avenida del charro 450 norte.

Código postal 32310 Ciudad Juárez, Chihuahua, México.

Tel: 6884800 al 09. • Fax: 6884813.

e-mail:mtescobe@uacj.mx; mayteesc@gmail.com

Recibido: 21 de Junio de 2014 / Aceptado: 29 de Septiembre 2014

"un estado emocional positivo que refleja una respuesta afectiva al trabajo"; sus variables pueden ser analizadas en el personal docente de las IES, dado que este tipo de entidades se han convertido en corporaciones abiertas y multinacionales ${ }^{5}$ provocando una diversificación en su cultura. En consecuencia, la integración de los Factores Socioculturales (FSC) se considera vital en el estudio y análisis de la SL.

Dado que las IES son instituciones en donde se requiere mejorar las estrategias para evaluar la SL y se encuentran diversos instrumentos de medición que consideran diferentes variables a las de interés para este estudio, se propone determinar y analizar los factores socioculturales (FSC) y ergoambientales (FEA) que puedan impactar en la satisfacción laboral de su personal académico, con la finalidad que las IES logren cumplir sus objetivos en materia de SL.

Para lo anterior, resulta necesario realizar un análisis de los instrumentos de medición existentes y evaluados por diversos autores y revisar las variables que contemplen los factores mencionados, estableciendo la integración o la falta de evaluación de las mismas, con lo que permitirá diseñar y proponer una escala de medición en la que se evalúe la relación entre la SL y los FSC y los FEA en el personal docente de las IES. 


\section{REVISION BIBLIOGRAFICA}

Diversos autores han evaluado la SL y su relación con varios factores, entre los que se encuentran las variables psicológicas, sociales, culturales, físicas, ambientales, etc. En el área de la psicología, Torres y Lajo ${ }^{6}$ determinan que tanto el estrés como el Síndrome de Desgaste Profesional (SDP) o Síndrome de Desgaste Ocupacional (SD0), conocido como bournout, influyen sobre la SL, proponiendo el replanteamiento de los programas educativos en el área de medicina por ser extenuantes.

López, Osca y Peiró ${ }^{7}$ presentan un estudio en donde relacionan los factores de estrés y la satisfacción laboral, analizando las seis fuentes de estrés en una escala que implica factores de trabajo, determinando que las variables psicológicas y las relacionadas con los sentimientos de deber y de obligación hacia el trabajo son elevados. Otro estudio que presentan Catalán y González ${ }^{8}$ sobre la actitud de los profesores hacia el proceso de evaluación de su docencia y los factores que se involucran en la SL en el momento de su autoevaluación, en el que se determinó que los profesores con una actitud positiva se autoevalúan mejor que los que tienen una actitud negativa, detectando en esta investigación que los factores que se analizan son los psicológicos en relación a SL.

En relación con variables sociales y culturales, los estudios siempre implican vinculación con conceptos y términos tales como ideología, comunicación, etnicidad, clases sociales, estructuras de pensamiento, género, nacionalidad, medios de producción y muchos otros que sirven para comprender los elementos únicos de cada comunidad, sociedad y etnia. ${ }^{9}$

La educación en general es parte fundamental dentro de los FSC, ya que puede adoptar o ignorar factores tan diversos como el ambiental, el social, etc. ${ }^{10}$ Estos también se relacionan con la valoración del trabajo. En este sentido se encuentran estadísticas en que los factores que más se relacionan en la SL son el horario de la jornada y la carga de trabajo. ${ }^{11}$

Otros factores a relacionar con la SL son los llamados ergo ambientales (EA). Estos se enfocan directamente en la interacción entre los usuarios y su ambiente físico. Este ambiente físico está caracterizado por la temperatura, el ruido, la vibración y la luminosidad $^{12}$, los mismos que vienen de la materia general de la ergonomía (del griego Ergon trabajo y Nomoi leyes naturales), la cual es el estudio de la optimización de la interfase entre los seres humanos y los objetos diseñados y el ambiente con los que ellos interactúan.

El valor de la ergonomía va más allá de la salud y la seguridad, ayudando a mantener dichos factores en los consumidores y trabajadores, añadiendo valor a la estrategia de negocios de la empresa para crear productos y servicios competitivos ${ }^{13}$, siendo que estos factores, así como la ergonomía, tienen relación con la evaluación de la fuerza de trabajo, el desempeño laboral, la satisfacción laboral y el diseño del ambiente de trabajo para maximizar la productividad y la salud, seguridad y bienestar de los trabajadores, en los que se hace hincapié. ${ }^{14}$ Pero la ergonomía ambiental es una parte integral de la disciplina de la ergonomía como ya se mencionó, y debe verse y practicarse desde esta perspectiva. Los humanos no responden al ambiente de manera monótona con relación a medidas directas del ambiente físico. Hay características humanas que determinan la sensibilidad y las respuestas de los humanos. ${ }^{15}$

En las investigaciones mencionadas anteriormente, se encuentran aspectos importantes como la problemática analizada y las reco- mendaciones propuestas. Entre los problemas que presentan las IES, como comentan Torres y Lajo ${ }^{7}$, están la falta de programas específicos que permitan al personal docente y/o administrativo manejar sus emociones. Catalán y González ${ }^{8}$ resaltan la falta de actitud entre los dirigentes de las mismas instituciones hacia su personal, sobrecargándolos de trabajo evitando su capacitación. Moreno et al. ${ }^{16}$ mencionan que la ausencia de diseños instruccionales y cursos de pedagogía merman el desempeño laboral provocando estrés y escasa remuneración.

De igual manera, las instituciones de educación superior carecen de programas que evalúen la SL y llevan de manera autónoma su propia evaluación al Desempeño Laboral de su personal como lo señalan Rueda et al. ${ }^{17}$ y en esta no contemplan dicho factor como lo es la SL. La evaluación de los estudiantes hacia el docente se puede llegar a ver influenciada tanto por factores personales como por el criterio y percepción de los mismos hacia los profesores, esto en la apreciación y análisis de Marginson ${ }^{1}$ y cuyos resultados pueden afectar o beneficiar la SL del docente. También se encuentra que los instrumentos de evaluación están mal elaborados y que las personas que los aplican y evalúan carecen de conocimientos en el área.

Los FSC influyen directamente en la autoevaluación del personal, siendo la edad y religión los que más impactan a la par de la actitud como lo exponen Díaz y Gallegos ${ }^{10}$ en su investigación, la misma que se refleja mediante la cultura y preparación de los empleados provocando, de acuerdo a las políticas internas de cada institución, que exista tensión en los mismos.

Relacionando la SL con los FEA, Parson ${ }^{13}$ expone las características humanas que corresponden a una reacción ante la Ergonomía Ambiental (EA), por lo que se recomienda en varios artículos, como el de Rabiull ${ }^{14}$ y el de Van Mechelen ${ }^{15}$, realizar estudios sobre los FEA en diferentes grupos de personas para determinar si impactan en la SL. De igual manera, considerar los FSC y económicos con los FEA y ver cómo se sienten los empleados.

\section{METODOLOGÍA}

Tomando como base el modelo conceptual propuesto de Escobedo et $a .^{18}$ en el que se analizan las variables estudiadas en diversas investigaciones, tales como los factores socioculturales y los factores ergoambientales y su impacto en la satisfacción laboral, se diseña un instrumento de medición dirigido al personal docente de las IES, para lo que es necesario analizar las metodologías existentes relacionadas a las variables en estudio.

\section{Métodos de evaluación de la Satisfacción Laboral}

Como ya se expuso, la SL es el sentir del individuo en su lugar de trabajo ${ }^{19}$, pero esto no se da porque sí. La SL tiene varios métodos e instrumentos que ayudan a la empresa y al mismo individuo a saber el grado (positivo o negativo) de SL en que se encuentran. Los métodos más conocidos para medir la SL son: La calificación única general y la calificación sumada, las cuales se conforman de varias partes del trabajo que se realiza ${ }^{20}$. El primer método consiste en un cuestionario sencillo con cinco posibles respuestas en escala de Likert, (también denominada Método de Evaluaciones Sumarias), que van desde "muy satisfecho" hasta "muy insatisfecho".

El segundo método es más elaborado ya que identifica los elementos clave de un trabajo y se pregunta al empleado su opinión respecto a cada uno de ellos. Este proceso evalúa factores 
específicos de índole laboral como la supervisión, el salario actual, oportunidades de ascender y la relación con los compañeros. ${ }^{21}$ Las respuestas se dan en una escala estandarizada y se suman para obtener una calificación general de la SL.

Robbins y Judge ${ }^{20}$ señalan que, hasta el momento se desconoce si un método es mejor que el otro, ya que investigaciones realizadas como la de Wanous, Reichers y Hudy ${ }^{22}$ tuvieron resultados semejantes con la aplicación de ambos métodos.

A pesar de ser sólo dos los métodos existentes en la evaluación de la SL, se encuentran estudios como el de Robles et $\mathrm{al}^{23}$ en que se utilizó el modelo europeo EFQM (European Foundation For Quality Management, por sus siglas en inglés), para analizar el grado de SL en el personal de un hospital en España. Este modelo contempla, en un 18\%, puntos de gestión de personal, con lo que se obtienen medidas de percepción que reflejan aspectos relacionados con la motivación y la satisfacción.

De igual manera, Tejero y Fernández ${ }^{24}$ crearon un instrumento para medir la satisfacción laboral llamado "Escala de Satisfacción Laboral en la Dirección Escolar", la que estima el grado de satisfacción en el que se encuentran los directivos en diversos aspectos de su trabajo, por medio de categorias que describen los motivos positivos o negativos, como resultado de su propia percepción. Este se maneja en una escala tipo Likert de 5 puntos que van desde 1 mucha insatisfacción y 5 mucha satisfacción y su estructura se conforma de cuatro fases. Como resultado se obtuvo un cuestionario con 25 preguntas.

Otro método de evaluación es el de Smith, Kendall y Hulin ${ }^{25}$ llamado Job Descriptive Index (J.D.I.) y en español se le conoce como Inventario de Satisfacción en el Trabajo (IST), el cual evalúa la SL con aspectos como: los compañeros, el trabajo y las tareas, las oportunidades de promoción, el mando y la satisfacción y, por último, el salario.

El Ministerio de Trabajo y Asuntos Sociales de España, a partir del 2000, creó la Encuesta de Calidad de Vida en el Trabajo (ECVT) en la que evalúa, entre otros factores, la SL. Ripoll et al. ${ }^{26}$ señalan que este instrumento permite medir el grado de satisfacción relacionado a diversos factores en cualquier ámbito, pertenecientes a la comunidad de Cataluña y de toda España.

Warr, Cook y Wall ${ }^{27}$ crearon la Escala de Satisfacción Laboral, la cual está formada por dos subescalas, siendo la SL total la suma de estas dos. La subescala de satisfacción laboral intrínseca y la extrínseca. La primera se refiere a aquellos aspectos que son consustanciales al trabajo: contenido del mismo, responsabilidad, logro, etc.; y la segunda, a las condiciones de trabajo en el sentido más amplio, tales como el salario, las políticas de la empresa, el entorno físico, la seguridad en el trabajo, etc. Según el modelo bifactorial de Herzberg et al. ${ }^{28}$, estos factores extrínsecos sólo pueden prevenir la insatisfacción laboral o evitarla cuando esta exista, pero no pueden determinar la satisfacción ya que esta estaría determinada por los factores intrínsecos.

\section{Comparativo de instrumentos de medición de la satisfacción laboral}

Dado que se encuentran varios instrumentos de medición respecto a la SL en la literatura del ramo, se analizan cuatro de estos para determinar cuáles ítems tienen más relevancia y si pertenecen a cualquiera de las variables contenidas en el modelo conceptual propuesto sobre SL.

Los cuestionarios a considerar son: la Escala General de Satisfacción (Overall Job Satisfaccion) creada por Warr, Cook y
Wall $^{27}$, la Escala de Satisfacción Laboral-Versión para profesores (ESL-VP) elaborada por Anaya y Suárez ${ }^{29}$, el Modelo de factores gestionables de Sánchez ${ }^{30}$ y la Escala de Satisfacción Laboral en la Dirección Escolar realizada por Tejero y Fernández. ${ }^{24}$ De estas anteriores se desprende la Tabla 1.

Las columnas mostradas en la Tabla 1 corresponden a las guías siguientes:

1. Instrumento

2. Percepción

3. Beneficios

4. Puesto

5. Reconocimiento

6. Relación con compañeros

7. Supervisión del jefe

8. Característicasfísicas del trabajo

9. Libertad de acción

10. Otros

Tabla 1.

Comparativo de instrumentos de medición de la satisfacción laboral.

\begin{tabular}{|c|c|c|c|c|c|c|c|c|c|}
\hline 1 & 2 & 3 & 4 & 5 & 6 & 7 & 8 & 9 & 10 \\
\hline $\begin{array}{l}\text { Warr, Cook } \\
\text { y Wall }{ }^{27}\end{array}$ & 7 & 10 & $\begin{array}{c}3 \\
12\end{array}$ & 4 & 3 & 5 & 1 & 2 & $\begin{array}{l}6 \\
9 \\
9\end{array}$ \\
\hline Anava y & 2 & 15 & 14 & 6 & 9 & 27 & 3 & 8 & 11 \\
\hline Suárez ${ }^{29}$ & 2 & $\begin{array}{c}5 \\
5 \\
8 \\
16 \\
8 \\
32 \\
19\end{array}$ & $\begin{array}{l}21 \\
25 \\
26\end{array}$ & $\begin{array}{c}7 \\
7 \\
9 \\
30 \\
31\end{array}$ & 10 & 29 & 3 & $\begin{array}{l}4 \\
15 \\
23 \\
28\end{array}$ & $\begin{array}{l}12 \\
13 \\
14 \\
22\end{array}$ \\
\hline Sánchez ${ }^{30}$ & 5 & $\begin{array}{c}2 \\
3 \\
6 \\
17 \\
13\end{array}$ & 4 & $\begin{array}{c}8 \\
16\end{array}$ & $\begin{array}{c}9 \\
15\end{array}$ & $\begin{array}{c}9 \\
14\end{array}$ & & & $\begin{array}{c}1 \\
7 \\
10\end{array}$ \\
\hline $\begin{array}{l}\text { Tejero y } \\
\text { Fernández }\end{array}$ & & $\begin{array}{c}1 \\
11\end{array}$ & $\begin{array}{l}9 \\
3 \\
2 \\
5 \\
6\end{array}$ & $\begin{array}{c}4 \\
10\end{array}$ & 8 & & & 7 & 12 \\
\hline
\end{tabular}

Fuente: Elaboración propia.

Los números observados de las columnas 2 a la 10, representan el número de ítem que corresponde al instrumento de medición de cada autor. Por ejemplo, Sánchez ${ }^{30}$ evalúa la variable "Percepción" con la pregunta cinco de su cuestionario y Anaya y Suárez ${ }^{29}$ con la pregunta dos. Para el mejor entendimiento de la tabla es necesario referirse a las guías mostradas con anterioridad.

Se observa en la tabla anterior que los ítems menos evaluados son los que corresponden a las variables percepción y condiciones físicas del trabajo. Respecto a la primera, esta se encuentra en tres de los cuatro cuestionarios analizados, siendo Tejero y Fernández ${ }^{24}$ los que no lo toman en cuenta. En relación a la segunda variable, sólo dos autores la analizan Warr, Cook y Wall ${ }^{27}$ y Anaya y Suárez $^{29}$. La supervisión del jefe es importante de analizar para los tres primeros autores. Pese a que los cuatro instrumentos consideran la relación con los compañeros, sólo un ítem lo evalúa en las encuestas de Anaya y Suárez ${ }^{29}$ y en la de Tejero y Fernández ${ }^{24}$. Para los primeros, la libertad de acción está analizada por cuatro items en el instrumento, no así en el de Sánchez ${ }^{30}$, en el que ni siquiera los menciona. El reconocimiento, desde el punto de vista de los cuatro autores, está dado por el clima afectivo, la formación del empleado, el esfuerzo, las promociones, el trabajo a la medida $\mathrm{y}$ el que sean tomadas en cuenta sus aportaciones. Y lo que 
respecta al puesto, se hace hincapié en factores como las horas trabajadas, el tiempo libre, las actividades y eficacia propias del puesto y la participación en proyectos innovadores, siendo que Sánchez ${ }^{30}$ sólo analiza el horario.

Como variable más estudiada está la de beneficios, en la que se analiza por medio de las promociones, en el caso de Warr, Cook y Wall $^{27}$ la estabilidad, seguridad, ausencia de presión, y las relaciones sociales por parte de Anaya y Suárez ${ }^{29} \mathrm{y}_{\text {de Sánchez }}{ }^{30}$. A su vez, Tejero y Fernández ${ }^{24}$ solo evalúan la colaboración y las oportunidades. Sin embargo, los cuatro autores miden factores que no están contenidas en las variables propuestas en el modelo conceptual de SL, siendo cuestiones políticas de gobierno y empresariales, como la imagen interna, las que más se repiten. La seguridad, el rendimiento y las necesidades y metas forman parte de este grupo. Como resultado del presente análisis, es necesario crear un instrumento que permita enfatizar las variables más relevantes de los autores mencionados, pero también robustecer las variables de condiciones físicas de trabajo, las cuales pertenecen al grupo de
FEA y la percepción o ingresos del empleado. Esta segunda permite detectar las carencias en cuanto a este rubro se refiere. Se toma el instrumento completo de Warr, Cook y Wall ${ }^{27}$ llamado "Escala General de Satisfacción (Overall Job Satisfaccion)" para medir la SL. Se integrarán items que permitan evaluar los FSC y los FEA en un nuevo instrumento de medición.

\section{Operacionalización de las variables}

A partir del modelo conceptual propuesto del impacto de los FSC y los FEA en la $\mathrm{SL}^{18}$, se presenta en la Tabla 2, tanto los constructos como las variables de medición y sus definiciones. En la última columna se observa el número de pregunta correspondiente al instrumento de medición resultante.

\section{Instrumento de medición}

El instrumento de medición "Escala de satisfacción laboral a partir de factores socioculturales y ergoambientales (ESL-FSCyFEA)", está conformado por 32 ítems, cuyos primeros quince pertenecen

Tabla 2.

Operacionalización de variables.

$\begin{array}{ll}\text { Constructo } & \text { Definición } \\ \text { Satisfacción Laboral } & \text { Es el conjunto de actitudes generales del individuo hacia su trabajo (Blum y Naylor, 1976). Conjunto de } \\ & \text { actitudes que tienen los trabajadores hacia su empleo (Robbins et al., 2009). Compromiso con el puesto } \\ \text { (el grado en que uno se identifica con su trabajo y participa activamente en él) y con la organización } \\ \text { (indicador de lealtad y la identificación con la empresa) (Atalaya, 1999). Valores que el individuo aplica } \\ \text { en sus diferentes facetas laborales (Flores, 1992). La comunicación abierta y positiva así como la } \\ \text { retroalimentación del jefe inmediato permite sentirse satisfecho en el trabajo (García y Ovejero, 2000). } \\ \text { La autonomia de planeación y acción aumenta la SL (Kittleson, 1996). Una relación amigable con los } \\ \text { compañeros, el obtener reconocimientos tanto económicos como profesionales y el conjunto de } \\ \text { beneficios que considera el empleado, son parte fundamental de mantenerse en un alto grado de SL } \\ \text { (Sánchez, 2008; Bedeian y Armenakis, 1981; Ostroff, 1992; Geber, 1991; Ward, 2001). Las condiciones } \\ \text { del trabajo enfatizan en lugares cómodos, ayudando a la proactividad (Márquez, 2006). La SL se verá } \\ \text { incrementada en relación a varios factores entre los que se encuentra la percepción. A mayor sueldo } \\ \text { mayor grado de SL (Puffer, 1987; Hogan y Hogan, 1989). }\end{array}$

Factores SocioCulturales

Factores Ergo-ambientales

Factores que interactúan entre los usuarios y su ambiente fisico (Greene, 2005). El ambiente físico se conforma por ruido (NOM-011-STPS-2001), lluminación (NOM-025-STPS-1999) y temperatura (NOM-015-STPS-2001). Exceso de ruido provoca malestares y dolores de cabeza resultando en un bajo rendimiento (Jara, 2008). Tanto temperaturas altas como bajas disminuye la ejecución de tareas (Tamez et al., 2003). La falta de iluminación natural y el exceso de iluminación artificial desencadena malestares oculares haciendo necesario el uso de anteojos (Guerrero et al., 2006).

\begin{tabular}{l|c|}
\hline Variable & No. item \\
\hline Percepción & 7 \\
\hline Beneficios & 1115 \\
\hline Puesto & 1314
\end{tabular}

Reconocimientos

Relación con compañeros

Supervisión del jefe

Condiciones de trabajo

Libertad de acción

Género

Edad

Religión

Lugar de nacimiento

Estado civil

Procedencia

Dependientes económicos

Último grado de estudios

Antigüedad laboral

Escuela de procedencia

Egresado de la misma

institución

Estudios actuales

Años de experiencia

professional

Ruido

32

Iluminación

Fuente: Elaboración propia. 
a Warr, Cook y Wall ${ }^{27}$, llamado "Escala General de Satisfacción (Overall Job Satisfacción)". Los siguientes trece corresponden a evaluar los FSC y los últimos cuatro corresponden a FEA.

La escala de medición utilizada generalmente es de cinco niveles (Likert, 193263). En este caso, Warr, Cook y Wall Warr, Cook y Wall ${ }^{27}$ propusieron una escala de siete niveles que permite robustecer el modelo propuesto. Esta va desde "muy insatisfecho" siendo el nivel uno o más bajo hasta "muy satisfecho" correspondiente al nivel 7 o más alto, y que ha sido utilizada por Moreno, Ríos, Canto, San Martín y Perles ${ }^{64}$, Alonso ${ }^{65}$, Rodríguez y Vetere ${ }^{66}$, Seoane, Garzón y Escamez ${ }^{67}$, entre otros.

Tabla 3.

Ítems del instrumento de SL a partir de FSC y FEA.

Constructo
Satisfacción Laboral
1. Condiciones fisicas de trabajo
2. Libertad para elegir tu propio método de trabajo
3. Tus compañeros de trabajo
4. Reconocimiento que obtienes por el trabajo bien hecho
5. Tu superior inmediato
6. Responsabilidad que se te ha asignado
7. Tu salario
8. La posibilidad de usar tus capacidades
9. Relaciones entre dirección y empleados de tu trabajo
10. Tus posibilidades de promoción
11. El modo en que tu institución está gestionada
12. La atención que se presta a las sugerencias que haces
13. Tu horario de trabajo
14. La variedad de tareas que realizas en tu trabajo
15. Tu estabilidad en el empleo
16. El género al que perteneces
17. La edad que tienes
18. La religión que profesas
19. El lugar donde naciste
20. Tu estado civil
21. El lugar de tu procedencia a esta ciudad
22. Tus dependientes económicos
23. Tu último grado de estudios
24. Tu antigüedad laboral
25. La escuela de procedencia
26. Si eres egresado de la misma escuela donde trabajas
27. Si estudias actualmente
28. Tu experiencia profesional
29. La iluminación natural en tu oficina
30, La iluminación artificial en tu oficina
31. La temperatura de tu oficina
32. El ruido fuera de tu oficina

Cabe mencionar que en el nuevo instrumento se diseñaron los textos que incluyen las variables analizadas por otros autores, por lo que no fue necesaria una traducción.

\section{RESULTADOS}

\section{Prueba piloto}

Siendo que la población a estudiar es de 1837 personas integrantes de cuatro IES privadas, la muestra obtenida mediante un software estadístico es de 157 con un nivel de confianza del 95\%. Para obtener el porcentaje de encuestas para aplicar en la prueba piloto y así validar el instrumento, se tomó la valoración de Ruíz et $a .^{68}$, en la que determina que debe ser del $10 \%$ de total de la muestra.

Se seleccionan cuatro universidades de las ocho totales pertenecientes a FIMPES en las que el personal académico y/o adminis- trativo contestará la encuesta, misma que identificará los factores socioculturales y ergoambientales y su impacto en la SL y cuyos datos se indican a continuación:

$$
\mathrm{n}=\frac{\delta N p q}{\mathrm{e}^{2}(\mathrm{~N}-1)+\delta^{2} p q}
$$

$\mathrm{N}$ : Total de la población a estudiar

$\delta$ : Nivel de confianza

e : margen de error

$p$ : probabilidad a favor

$q$ : probabilidad en contra

Correspondiente al presente análisis, los valores se consideran como siguen:

$\mathrm{N}=1837$ personas

$\delta=1,96 \quad$ (intervalo de confianza al $1-\alpha=95 \%$ )

e $=6 \% \quad$ (margen de error)

$\mathrm{p}=80 \% \quad$ (probabilidad a favor)

$q=20 \% \quad$ (probabilidad en contra)

Para determinar el tamaño de la población, se tomó como referencia los datos obtenidos através del departamento de recursos humanos, por lo que se determina la muestra como se indica:

$$
\mathrm{n}=\frac{1,96(1837)(0,8)(0,2)}{(0,6)^{2}(1837-1)+(1,96)^{2}(0,8)(0,2)}=157
$$

Para realizar el análisis de esta investigación, la prueba piloto señaló que los instrumentos a aplicar son 16; sin embargo,se intentó realizar a 30 personas, quedando al final 26 contestadas. De las anteriores, se analizó la validez de todo el instrumento por medio de Alpha de Crombach dando como resultado 0,941 a los 32 ítems (Tabla 4).

De este primer análisis se evaluaron 18 encuestas (69,2\%), excluyendo $8(30,8 \%)$ en los que los encuestados dejaron en blanco alguna de las casillas (Tabla 5).

Tabla 4.

Valor Alpha de Crombach de los 32 ítems. Estadísticaconfiable.

$\begin{array}{cc}\text { Alpha de Crombach } & \text { No de items } \\ 0,941 & 32\end{array}$

Tabla 5.

Validación y exclusión de encuestas en la prueba piloto.

\begin{tabular}{|crcc}
\multicolumn{4}{c}{ Resumen de procesamiento de casos } \\
Casos & N & $\%$ \\
\cline { 2 - 4 } & Válidos & 18 & 69,2 \\
& Excluidosa & 8 & 30,8 \\
& Total & 26 & 100,0
\end{tabular}

a. Eliminación por lista basada en todas las variables del procedimiento.

Tabla 6.

Escala de coeficiente de confiabilidad.

$\begin{array}{cc}\text { Rangos } & \text { Magnitud } \\ 0,81 \text { a } 1,00 & \text { Muy alta } \\ 0,61 \text { a } 0,80 & \text { Alta } \\ 0,40 \text { a } 0,60 & \text { Moderada } \\ 0,21 \text { a } 0,40 & \text { Baja } \\ 0,01 \text { a } 0,20 & \text { Muy baja }\end{array}$

Fuente: Elaboración propia. 
Considerando el resultado anterior, se determina que la escala es altamente confiable a un nivel de significancia de 0,05, tomando el coeficiente de confiabilidad de la siguiente escala ${ }^{68}$. Ver Tabla 6.

\section{Validez y confiabilidad}

$\mathrm{Al}$ analizar cada uno de los ítems del instrumento piloto, se toma la validez individual a partir de 0,33 por lo que se determina que el ítem 17, 19, 20, 21, 27 y 28 con 0,218, 0,218, 0,105, 0,198, 0,018 y 0,306 respectivamente, se deben eliminar. Ver Tabla 7.

$\mathrm{Al}$ evaluar el instrumento completo eliminando los ítems mostrados anteriormente, el coeficiente de correlación cambia de 0,941 a 0,948 (ver Tabla 8). Considerando que la diferencia es mínima, se determina hasta el momento que se aplique el instrumento original.

\section{Coeficiente de correlación de la satisfacción laboral}

$\mathrm{Al}$ evaluar el instrumento por ítems correspondientes a los constructos, se obtiene que, en lo relacionado a la SL a la que pertenecen los primeros 15 items del instrumento, su coeficiente de correlación es de 0,926 (Tabla 9), determinando que sólo la pregunta 3 debe

Tabla 7.

Validación del instrumento piloto.

\begin{tabular}{|c|c|c|c|c|}
\hline & Estadis & ca total por íte & & \\
\hline & $\begin{array}{r}\text { Escala media } \\
\text { de elementos } \\
\text { eliminados }\end{array}$ & $\begin{array}{l}\text { Escala de } \\
\text { varianza de } \\
\text { elementos } \\
\text { eliminados }\end{array}$ & $\begin{array}{l}\text { Correlación } \\
\text { por item }\end{array}$ & $\begin{array}{l}\text { Alpha de } \\
\text { Cronbach } \\
\text { si el item } \\
\text { se elimina }\end{array}$ \\
\hline item1 & 177,78 & 430,301 & 0,513 & 0,940 \\
\hline item2 & 178,17 & 405,794 & 0,792 & 0,937 \\
\hline 13 & 178,17 & 425,676 & 0,346 & 0,942 \\
\hline 14 & 179,06 & 391,467 & 0,805 & 0,936 \\
\hline 15 & 178,28 & 414,683 & 0,508 & 0,940 \\
\hline 16 & 177,83 & 423,441 & 0,639 & 0,939 \\
\hline 17 & 178,83 & 402,853 & 0,709 & 0,938 \\
\hline 18 & 178,50 & 410,853 & 0,508 & 0,940 \\
\hline 19 & 178,56 & 406,497 & 0,674 & 0,938 \\
\hline 110 & 178,78 & 425,712 & 0,386 & 0,941 \\
\hline |11 & 178,22 & 420,889 & 0,685 & 0,939 \\
\hline 112 & 178,56 & 406,144 & 0,777 & 0,937 \\
\hline 113 & 178,28 & 404,801 & 0,843 & 0,937 \\
\hline I14 & 178,00 & 404,471 & 0,912 & 0,936 \\
\hline 115 & 178,44 & 409,673 & 0,583 & 0,939 \\
\hline 116 & 177,67 & 418,353 & 0,577 & 0,939 \\
\hline 117 & 177,33 & 437,059 & 0,218 & 0,942 \\
\hline I18 & 177,56 & 418,732 & 0,687 & 0,939 \\
\hline 119 & 177,33 & 437,059 & 0,218 & 0,942 \\
\hline 120 & 177,44 & 439,438 & 0,105 & 0,943 \\
\hline 121 & 177,61 & 435,546 & 0,198 & 0,942 \\
\hline 122 & 177,89 & 419,752 & 0,527 & 0,940 \\
\hline 123 & 178,06 & 414,056 & 0,749 & 0,938 \\
\hline 124 & 178,11 & 413,516 & 0,805 & 0,938 \\
\hline 125 & 177,72 & 428,918 & 0,347 & 0,941 \\
\hline 126 & 178,28 & 412,565 & 0,598 & 0,939 \\
\hline 127 & 178,00 & 442,706 & 0,018 & 0,945 \\
\hline 128 & 177,67 & 433,176 & 0,306 & 0,941 \\
\hline 129 & 178,33 & 402,824 & 0,691 & 0,938 \\
\hline 130 & 178,44 & 400,144 & 0,696 & 0,938 \\
\hline |31 & 178,78 & 397,830 & 0,684 & 0,938 \\
\hline 132 & 178,89 & 395,516 & 0,762 & 0,937 \\
\hline
\end{tabular}

Tabla 8.

Valor de Alpha de Crombach sin ítems debajo de 0,33.

\begin{tabular}{|c|c|c|c|}
\hline \multicolumn{4}{|c|}{ Estadística confiable } \\
\hline & $\begin{array}{c}\text { Alpha de Cronbach } \\
0,948\end{array}$ & $\mathrm{~N}^{0} \mathrm{~d}$ & \\
\hline \multicolumn{4}{|c|}{ Escala estadística } \\
\hline Media & Varianza & Desv. estándar & Node ítems \\
\hline 183,89 & 443,046 & 21,049 & 32 \\
\hline
\end{tabular}

descartarse ya que mide 0,257 estando por debajo de 0,333, valor tomado como mínimo para determinar si una pregunta se descarta o no. Sin embargo, dado el resultado de correlación, se considera dejarla ya que no afecta el nivel de confiabilidad. (Ver Tabla 10).

\section{Coeficiente de correlación de los factores socioculturales}

$\mathrm{Al}$ evaluar el instrumento en los ítems correspondientes a los factores socioculturales, los cuales pertenecen a las preguntas 16 a la 28, trece en total, se determina que su coeficiente es de 0,836 (Tabla 11).

En este análisis se detecta que únicamente el item 27 debe ser eliminado por obtener un resultado de 0,007.

Tabla 9.

Alpha de Crombach de los ítems de satisfacción laboral.

\begin{tabular}{cc}
\multicolumn{3}{c}{ Estadística confiable } \\
Alpha de Cronbach & $N^{0}$ de items \\
0,926 & 15
\end{tabular}

Tabla 10.

Estadística general de los ítems de satisfacción laboral.

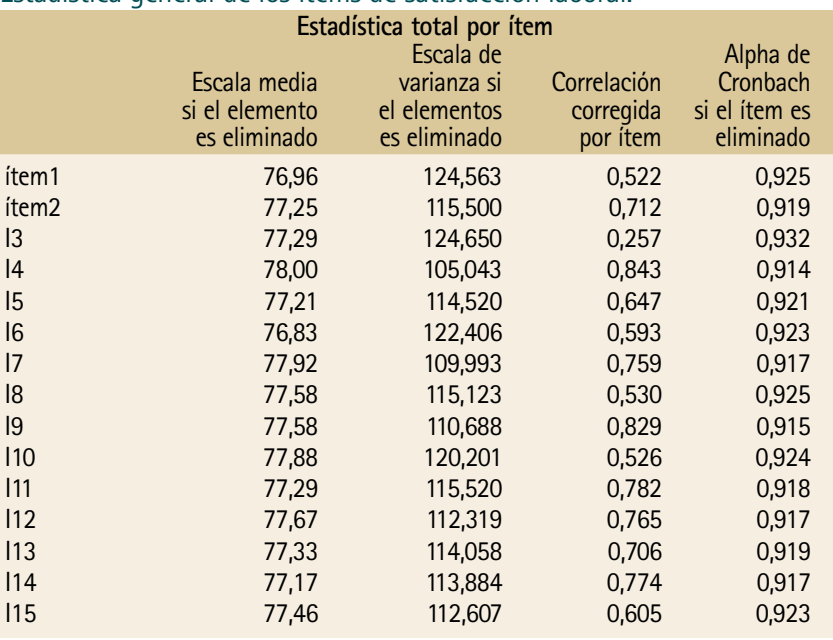

Tabla 11.

Correlación de los ítems de factores socioculturales.

$\begin{array}{cc}\text { Alpha de Cronbach } & N^{0} \text { de items } \\ 0,836 & 13\end{array}$

Tabla 12.

Estadística general de los ítems de factores socioculturales.

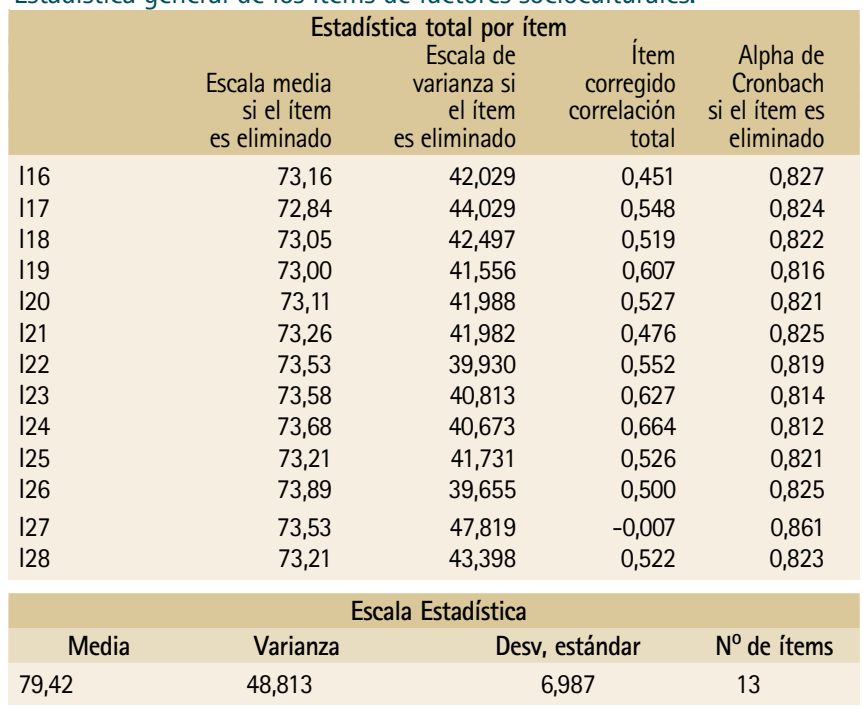


Tabla 13.

Alpha de Crombach para factores ergoambientales.

$\begin{array}{cc}\text { Alpha de Cronbach } & \text { Node items } \\ 0,955 & 4\end{array}$

Tabla 14.

Estadistica general de factores ergoambientales.

\begin{tabular}{|c|c|c|c|c|}
\hline \multicolumn{5}{|c|}{ Estadistica total por ítem } \\
\hline & $\begin{array}{l}\text { Escala media } \\
\text { si el item } \\
\text { es eliminado }\end{array}$ & $\begin{array}{r}\text { Varianza si } \\
\text { el item } \\
\text { es eliminado }\end{array}$ & $\begin{array}{r}\text { İtem } \\
\text { correcto- } \\
\text { correlación } \\
\text { total }\end{array}$ & $\begin{array}{r}\text { Alpha de } \\
\text { Cronbach } \\
\text { si el item es } \\
\text { eliminado }\end{array}$ \\
\hline 129 & 16,00 & 16,583 & 0,832 & 0,958 \\
\hline 130 & 16,12 & 14,943 & 0,933 & 0,927 \\
\hline 131 & 16,48 & 14,427 & 0,883 & 0,944 \\
\hline 132 & 16,56 & 14,673 & 0,920 & 0,931 \\
\hline \multicolumn{5}{|c|}{ Escala Estadística } \\
\hline Media & Varianza & & estándar & $\mathrm{N}^{0}$ de ítems \\
\hline 21,72 & 26,543 & & 152 & 4 \\
\hline
\end{tabular}

\section{Coeficiente de correlación de los factores ergoambientales}

El valor del alpha de Cronbach para los factores ergoambientales es de 0,955 (ver Tabla 13), correspondiente a cuatro items, En este caso se aceptan todas las preguntas dado que el resultado de la correlación es mayor a 0,832 (Tabla 14).

\section{Instrumento final}

Tomando en cuenta los valores anteriores, se determinó que el instrumento utilizado en la prueba piloto se aplique con los 32 items originales.

\section{CONCLUSIONES Y RECOMENDACIONES}

Se analizaron instrumentos de medición que evalúan la SL a través de variables pertenecientes a diferentes ámbitos. Aquellos que analizan tanto parcial como totalmente las variables contenidas en el presente estudio, se evaluaron y compararon, ayudando a diseñar y analizar el instrumento propio y particular para los fines de esta investigación,

Las variables en estudio coinciden, por la SL, con Wanous et al. ${ }^{22}$, Robles et al. ${ }^{23}$, Armas ${ }^{69}$, Briseño et al. ${ }^{70}$, Gálvez et al. ${ }^{71}$, Falcón et al. ${ }^{72}$, entre otros.

Respecto a las variables socioculturales, en el presente estudio se agregan a las estudiadas con anterioridad por otros investigadores las correspondientes al último grado de estudio, la antigüedad laboral, la escuela de procedencia y la relación con los compañeros de trabajo. El resto de las variables se agregan al instrumento por no considerarse en otros estudios.

Las variables contenidas en los FEA analizadas con anterioridad se analizan en el presente, agregando la variable iluminación natural y eliminando la variable vibración por no tener significancia en el trabajo realizado por el docente.

La escala de medición resultante del análisis y la comparación de instrumentos utilizados por otros investigadores tiene una confiabilidad de 0,941, por lo que se decide dejar la original ya que de eliminar aquellas variables con valor menor a 0,33 el valor de confiabilidad queda de 0,948, siendo casi imperceptible.

Se recomienda aplicar la encuesta en diversas IES para determinar las variables que tienen mayor o menor relación con la SL, eliminando en la prueba piloto aquellas que se consideren con significancia baja.

\section{REFERENCIAS}

1. Marginson, $S$, The knowledge economy and higher education: a system for regulating the value of knowledge, Innovación Educativa, 2009; 9(47), Recuperado de http://redalyc,uaemex,mx/src/inicio/ArtPdfRed,jsp?iCve=179414895 007\&iCveNum $=14895$

2. Locke, $E$, The Ubiquity of Technique of oal Setting in Theories of and Approaches to Employee Motivation, Academy Management Review,1976; 594-601.

3. González, M, Hernández de Rincón, $A$, y Hernández, A, Desempeño de alumnos y docentes de matemática desde el constructivismo, Caso Algebra Lineal, Facultad de Ingenieria, LUZ, Multiciencias, 2007; 7(3): 282-292,

4. Belfield, C, Harris, R, How well do theories of job matching explain variations in job satisfaction across education levels? Evidence for UK graduates, Applied Economics, 2002;34; 535-548.

5. Brunner, J, Educación superior en América Latina: Cambios y Desafios, Fondo de Cultura Económica, Santiago de Chile 1990.

6. Torres, M,Lajo, $R$, Variables psicológicas implicadas en el desempeño laboral docente, Revista IIPSI, Facultad de psicología, UNMSM,2008 Dic; 11(2), Recuperado de $h$ ttp://www,scielo,org,pe/pdf/rip/v11n2/a08v11n2,pdf

7. López, B, Osca, A,Peiró, J, El papel modulador de la implicación con el trabajo en la relación entre el estrés y la satisfacción laboral, Psicothema,2007; 19(1), Recuperado de http://dialnet,unirioja,es/servlet/articulo?codigo=2231550

8. Catalán, J, y González, M, Actitud hacia la Evaluación del Desempeño Docente y su Relación con la Autoevaluación del Propio Desempeño, en Profesores Básicos de Copiapo, La Serena y Coquimbo, Psykhe, 2009; 18(2): 97-112.

9. Nanda, S, Antropología cultural, adaptaciones socioculturales, Iberoamericana, 1987; 80-96: 117-118.
10. Diaz, O, Gallegos, R,Formación y práctica docente en el medio rural, PyV, 1996; 122-131.

11. Clark, B,El Sistema de educación superior: Una visión comparativa de la organización académica, Nueva Imagen, Universidad Autónoma Metropolitana, Universidad Futura, México, 1991.

12. Kompier, M, Geurts, A, Gründemann, W, Vink, P, y Smulders, G, Julio, Cases in stress prevention: The success of a participative and stepwise approach, Stress, Journal of Medicine, 1998; 14(3):155- 168, Recuperado de http://www,ingenta connect,com/content/jws/smi/1998/00000014/00000003/art00773

13. Parsons, $K, C$, La ergonomía ambiental: Una revisión de principios, métodos $y$ modelos, Applied Ergonomics, 2000; 31(6): 581 -594.

14. Rabiul, A, Occupational health, safety and ergonomicissues in small and medium-sized enterprises in a developing country, Department of Process and Environmental Engineering and Work Science Laboratory, University of Oulu, Oulu, 2002.

15. Van Mechelen, $\mathrm{W}$, Occupational health, safety and ergonomic issues in small and médium-sized enterprises in a developing country, Journal Occupational Ergonomics, 2007;7(1): 23-36.

16. Moreno, R, Cepeda, I, y Romero, S, El modelo de evaluación, intervención y análisis de procesos como propuesta de diseño instruccional, Enseñanza e Investigación en Psicologia, 2004; 9(2). Recuperado de http://redalyc,uaemex, $\mathrm{mx} / \mathrm{pdf} / 292 / 29290205, \mathrm{pdf}$

17. Rueda, B, Elizalde, L, y Torquemada, G, La evaluación de la docencia en las Universidades mexicanas, Revista de la Educación Superior, 2003 Julio; 32(3), Recuperado de Http://www,anuies,mx/servicios/p_anuies/publicaciones/ revsup /127/02e,html 
18. Escobedo, M, Cuautle, L, Sánchez, G, Cavazos, J,Maynez, A, Factores Socioculturales y Ergoambientales: su impacto en la Satisfacción Laboral de las Instituciones de Educación Superior, Teoria y Praxis, 2013;14:83-108.

19. Hodson, R, Workplace Behaviors, Work and Occupations, 1991; 271-290.

20. Robbins, S, Judge, T, Comportamiento Organizacional, 13a ed, Prentice Hall, México, 2009.

21. Spector, E, Job Satisfaction: Application, Assessment, Causes and Consequences, Thousand Oaks, California, 1997.

22. Wanous, J, Reichers, A,Hudy, M, Overall, Job Satisfaction: How Good are SingleItem Measures?,Journal Applied Psychology, 1997; 247-252.

23. Robles, M, Dierssen, $T$, Martínez, E, Herrera, $P$, Díaz, A,Llorca, J, Variables relacionadas con la Satisfacción Laboral: un estudio transversal a partir del modelo EFOM, GacSanit, 2005; 19(2): 127-34.

24. Tejero, $C$, Fernández, M, Medición de la Satisfacción Laboral en la Dirección Escolar, Relieve, 2009; 15 (2):1-16.

25. Smith, P, Kendall, L, Hulin, C, The measurement of Satisfaction in Work and Retirement: A strategy for the study of attitudes, Rand McNally, Chicago, 1969.

26. Ripoll, E, Falguera, $M$, Urrutia, J, Factores determinantes de la satisfacción en el trabajo: cinco años del barómetro laboral, Índice, 2006; 14 -16.

27. Warr $\mathrm{P}$, Cook J, Wall $\mathrm{T}$, Scales for the measurement of some work attitudes and aspects of psychological well-being, Journal of Occupational psychochology, 1979; 52: 11-28.

28. Herzberg, F, Mausner, B, Snyderman, B, The motivation to work, 2nd ed, Editorial John Wiley and Sons, New York, 1959.

29. Anaya, D, Suárez, J, Satisfacción laboral de los profesores de educación infantil, primaria y secundaria, Un estudio de ámbito nacional, Revista Educación, 2007;344:217-218.

30. Sánchez, C, Motivación, Satisfacción y Vinculación, ¿Es Gestionable la Voluntad de las Personas en el Trabajo? Acción Psicológica, 2008; 5(1): 9-28.

31. Blum, M, Nylor, J, Psicología Industrial,1a ed, Ed, Trillas, Mexico, 1976.

32. Atalaya, M, Satisfacción laboral y productividad, Revista Psicologia, 1999; 3(5): 46-56.

33. Bedeian, A, Armenakis, A, A Path-Analytic Study of the Consequences of Role Conflict and Ambiguity, Academy Management Journal, 1981 Junio; 24(2): 417-424.

34. Flores, $\mathrm{R}, \mathrm{El}$ comportamiento humano en las organizaciones, Universidad del Pacífico, Lima, 1992.

35. Garcia, A, I, Ovejero B, Feedback Laboral y Satisfacción, Universidad de Oviedo, España, 2000.

36. Kittleson, Ch, Identifying and Correcting Unsafe Employee Attitudes and Behavior, Supervisor Safety Update, EEUU, 1996.

37. Bedeian, A, Armenakis, A, A Path-Analytic Study of the Consequences of Role Conflict and Ambiguity, Academy management Journal,1981; 24(2): 417-424.

38. Ostroff, C, The Relationship between Satisfaction, Attitudes and Performance: An Organizational Level Analysis, Journal of Applied Psychology, 1992; 963-74.

39. Geber, B, The Bugaboo of Team Pay, Training, 1995; 32(8): 25-34.

40. Ward, E, Social Power Bases of Managers: Emergence of a New Factor, Journal Social Psychology, 2001; 144-47.

41. Márquez, M, Satisfacción laboral, Comunidad Latina de estudiantes de negocios, Gestio Polis, 2002. Recuperado de: http://www,gestiopolis,com/recursos/ documentos/fulldocs/rrhh/satlab,htm

42. Puffer, S,Prosocial Behavior, Noncompliant Behavior and Work Performance among Commission Salespeople, Journal Applied Psychology, 1987; 615-21.

43. Hogan, J, Hogan, R, How to measure Employee Reliability, Journal Psychology, 1989; 273-279.

44. Bosque, J, Vilá, J, Geografia de España, Geografia humana, Ed, Planeta, 1á, Ed, España, 1992; 115-127.

45. Sánchez, A, El Sentido de la Vida, Revista Humanidades Médicas,2005; 5(1).

46. Montiel, E, El nuevo orden simbólico: la diversidad cultural en la era de la globalización, Lit, lingüistica, 2003; 14.

47. Otto, I, Sans, M, Montiel, M, Enfermeria Médico Quirúrgica, Segunda Edición: Necesidad de nutrición y eliminación, Editorial Masson, 2003; 64-78.

48. Sastre, V, La cultura del ocio, Implicaciones sociales y eclesiásticas del fin de semana, Universidad Pontifical de Madrid, 2005; 112-132.
49. Ramírez, J, Cubero, M, SantaMaria, A, Cambio sociocognitivo y organización de las acciones: una aproximación sociocultural a la educación de adultos, Journal Study Education and Development, 1990; 169-190.

50. Gamero, C, Satisfacción laboral de los asalariados en España, Especial referencia a las diferencias por género, Cuadernos de economía, 2004;27: 109-146.

51. Guzmán, T, Sánchez, $S$, Nascimento, $M$, Gomes, $A$, La satisfacción laboral del capital humano femenino en la actividad hotelera, Un estudio de caso para Andalucía y Algarve, Revista Universitaria Ciencias Trabajo, 2010; 127-145.

52. Escudero, J, Delfín, L, Ladrón de Guevara, R, Estudio del Sindrome Quemado por el Trabajo (Burnout) en el Instituto Tecnológico Superior de Xalapa-Enríquez, Simposio Internacional de Campos Emergentes en la Formación de Profesionales de la educación, 2008 Nov 6-8, Mazatlán, Sinaloa, México.

53. Álvarez-Flores, D, Satisfacción y fuentes de presión laboral en docentes universitarios de Lima metropolitana, 2007; 10: 49-97. Recuperado en: http://www, redalyc,org/articulo,oa?id $=147112813004$

54. López, F, Bernal, L, Cánovas, A, Satisfacción laboral de los profesionales en un Hospital Comarcal de Murcia, Revista Calidad Asistencial,2001; 16:243-246.

55. Galaz, J, La satisfacción laboral de los académicos en una universidad estatal pública, Revista Perfiles Educativos, 2002; 24(96): 47-52.

56. Greene, $B, L$, Dejoy, D, M, Olejnik, $S$, Effects of an active ergonomics training program on risk exposure, worker beliefs, and symptoms in computer users, Journal Work, 2005; 24(1): 41-52.

57. Normas oficinales mexicanas 011-STPS-2001, Condiciones de seguridad e higiene en los centros de trabajo donde se genere ruido, D, 0, F, 17 IV (2002).

58. Normas oficinales mexicanas 025-STPS-2008, Condiciones de iluminación en los centros de trabajo, D, O,F, 20 XII (2008).

59. Normas oficinales mexicanas 015-STPS-2001, Condiciones térmicas elevadas 0 abatidas, Condiciones de seguridad e higiene, D, 0, F, 14 VI (2002).

60. Jara, F, El estrés laboral y la ecología emocional, Manual de psicología jurídica laboral, Publicaciones universitarias, Madrid, 2008.

61. Tamez, S, Ortiz, L, Martínez, S, Méndez, I, Riesgos y daños a la salud derivados del uso de videoterminal, Revista Salud Pública México, 2003; 45(3).

62. Guerrero, J, Cañedo, R, Rubio, S, Cutiño, M, Fernández, D, Calidad de vida y trabajo, Algunas consideraciones sobre el ambiente laboral de la oficina, ACIMED, 2006; 14(4).

63. Cañadas, I, Sánchez, A, Categorias de respuesta en escalas tipo Likert, Psycothema, 1998; 10(3): 623-631.

64. Moreno, M, Ríos, L, Canto, J, San Martín, J, Perles, F, Satisfacción laboral y Burnout en trabajos poco cualificados: diferencias entre sexos en población inmigrante, Revista de Psicología del Trabajo y de las Organizaciones, 2010; 26(3):255-265,

65. Alonso, P, Diferencias en la percepción de la satisfacción laboral en una muestra de personal administrativo, Boletín de Psicología, 2006 Nov; 88: 49-63.

66. Rodriguez, R, Vetere, G, Adaptación Argentina del cuestionario de creencias obsesivas, Interdisciplinaria, 2008; 25(1).

67. Seoane, J, Garzón, A, Escámez, J, Análisis de la Aplicación de la LOGSE, Opiniones de diversos Sectores Sociales, IVECE, Consejeria de Educación, Generalitat Valenciana, 2000 Mayo-diciembre.

68. Ruiz, M, Pardo, A, San Martin, R, Modelos de ecuaciones estructurales, 2010; 31(1): 34-45.

69. Armas, M, Evaluación de la satisfacción laboral, estrés y autoestima de los directivos escolares, Dirección participativa y evaluación de centros, Actas del II Congreso Internacional sobre Dirección de Centros Docentes,ICE de la Universidad de Deusto,Bilbao, 1996; 419-429.

70. Briseño, $C$, Herrera, $R$, Enders, J, Fernández, $A$, Estudio de riesgos ergonómicos y satisfacción laboral en el personal de enfermeria, Revista de la escuela de salud pública, 2005; 9(1): 53-59.

71. Gálvez, L, Ponce, $M, 2005$, Condiciones de riesgo ergonómico en los administrativos de una institución de educación superior en Pereira, Investigaciones Andina Redalyc, 7(10), 5-9.

72. Falcón, A, Díaz, L, Variables que predicen la satisfacción y la motivación de los directores de organizaciones educativas, Avances de Supervisión Educativa,2007; 6 . 